\title{
NOUVELLE
}

\section{Pseudomonas aeruginosa utilise ses bactériophages filamenteux pour manipuler la réponse immunitaire}

Mathieu De Jode ${ }^{1,2}$, Laurent Debarbieux ${ }^{1}$

\author{
${ }^{1}$ Institut Pasteur, groupe interactions bactériophages- \\ bactéries chez l'animal, département de microbiologie, \\ 25 , rue du Docteur Roux, 75015 Paris, France. \\ ${ }^{2}$ Sorbonne université, collège doctoral, F-75005 Paris, France. \\ laurent.debarbieux@pasteur.fr
}

> Pseudomonas aeruginosa, ou bacille pyocyanique, est une bactérie Gramnégative responsable d'infections diverses (pulmonaires, urinaires, cutanées, etc.), qui sont d'autant plus sévères que cette bactérie est devenue plus résistante aux antibiotiques. L'Organisation mondiale de la santé a d'ailleurs récemment classé $P$. aeruginosa comme un pathogène prioritaire, contre lequel il est urgent de découvrir de nouveaux traitements. La virulence de $P$. aeruginosa repose sur de nombreux facteurs, tels que toxines, flagelle ou pilus, mais dans un article récemment publié dans Science, Sweere et al. ont montré que $P$. aeruginosa utilisait un nouveau type de facteur de virulence: le bactériophage filamenteux Pf4, qui réduit la réponse immunitaire dirigée contre cette bactérie [1].

\section{Le bactériophage filamenteux Pf4}

Les bactériophages filamenteux sont des bactériophages tempérés ${ }^{l}$, dont les virions, constitués de protéines structurales enrobant le génome viral (ADN simple brin), ressemblent à de longs tubes (6 à $8 \mathrm{~nm}$ de diamètre et 800 à $2000 \mathrm{~nm}$ de longueur). Contrairement aux bactériophages virulents utilisés en phagothérapie, qui lysent rapidement les bactéries qu'ils infectent, les bactériophages filamenteux sont sécrétés par la bactérie sans la détruire [2]. Les auteurs avaient précédemment décou-

${ }^{1}$ Un bactériophage tempéré qualifie un phage dont le génome peut s'intégrer dans l'ADN de la bactérie hôte et en transformer les propriétés. vert que les bactériophages filamenteux de $P$. aeruginosa perturbaient la réponse inflammatoire, et étaient ainsi impliqués dans la virulence de ce pathogène [3]. Les bases mécanistiques de l'effet anti-inflammatoire du bactériophage Pf4 sont rapportées dans leur nouvel article.

\section{Le phage filamenteux Pf4 est} important pour la colonisation de l'organisme hôte par $P$. aeruginosa Afin d'explorer le rôle du bactériophage Pf4 dans l'infection par P. aeruginosa, les chercheurs ont supprimé l'ADN de ce bactériophage du génome de la souche bactérienne PAOl. Ils ont ainsi pu découvrir, sur un modèle murin d'infection, que les souches qui possèdent l'ADN de Pf4 colonisent mieux une plaie ouverte que les souches qui en sont dépourvues. De plus, les infections par la souche possédant I'ADN de Pf4 sont caractérisées par une augmentation de la morbidité et de la mortalité des animaux par rapport aux infections par la souche qui en est dépourvue. Pour expliquer cette augmentation de la virulence de $P$. aeruginosa contenant le génome de Pf4, les auteurs ont analysé les effets de Pf4 sur le système immunitaire de l'organisme hôte.

Pf4 active une réponse anti-virale qui diminue la réponse anti-bactérienne

Le recrutement des cellules immunitaires sur le site de l'infection n'est pas affecté par la présence du bactériophage, mais le nombre de bactéries internalisées par des cellules dendri- tiques murines ou des macrophages murins et humains est réduit en présence de Pf4. Cependant, la destruction des bactéries par phagocytose n'est pas altérée, ce qui signifie que Pf4 agit sur l'étape de capture de la bactérie par le macrophage. Les auteurs ont découvert que la production de TNF- $\alpha$ (tumor necrosis factor alpha) par des macrophages, en réponse à une stimulation de la réponse inflammatoire par l'ajout de lipopolysaccharide (LPS) bactérien, est diminuée en présence de Pf4. Cette diminution est due à une induction de la production d'interférons de type I (IFN-I), un marqueur de la réponse immunitaire antivirale, stimulée par la présence du bactériophage Pf4. Les auteurs ont ensuite montré que cette activation de la production d'IFNl s'effectuait via TRIF (TIR-domain-containing adapterinducing interferon- $\beta$ ), lui-même activé par TLR3 (Toll-like receptor 3 ), dont la fonction est notamment de reconnaître les molécules d'ARN double brin.

Des molécules d'ARN du bactériophage Pf4 ont ensuite été produites et purifiées afin de montrer qu'elles sont capables d'activer TLR3 exprimé par des monocytes humains. Étonnamment, des ARN correspondant à des gènes du bactériophage Pf4 ont également été détectés dans les cellules humaines incubées avec celui-ci. Cela suggère que soit I'ADN de ce bactériophage est transcrit dans les cellules eucaryotes, soit le phage relargue des molécules d'ARN encapsidées lors de la formation des virions. Aucune de ces deux hypothèses n'est explorée dans l'article, mais elles feront 


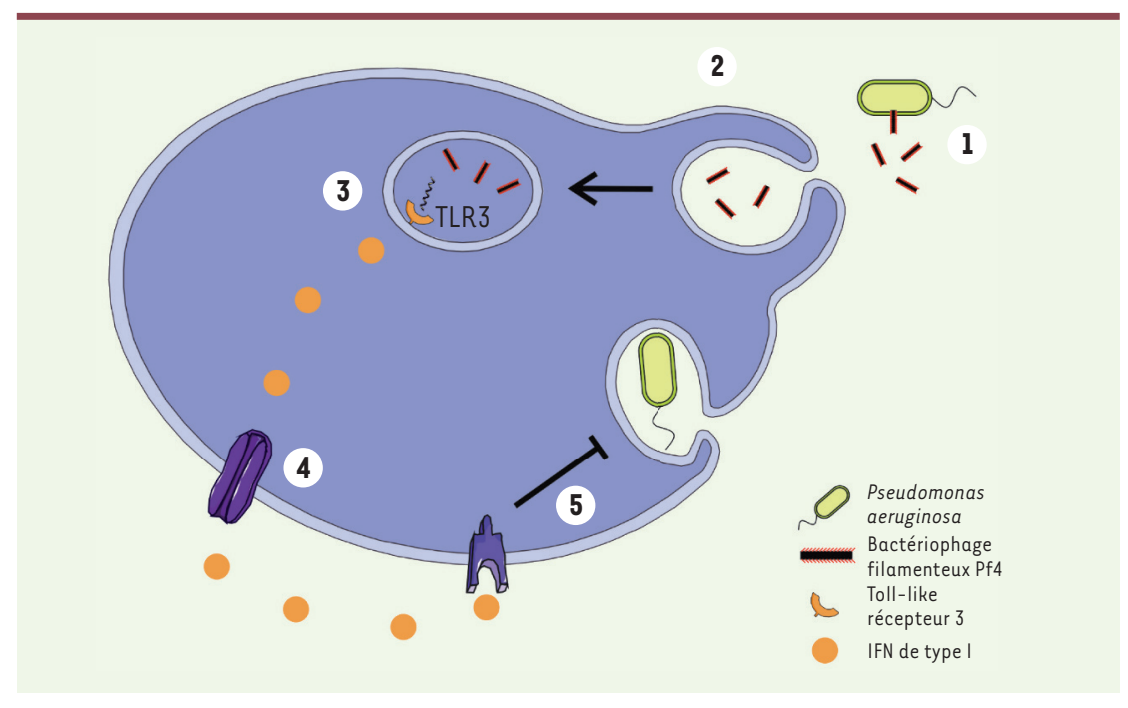

sans aucun doute l'objet de futurs travaux de recherche.

Les auteurs ont ensuite marqué le bactériophage Pf4 par un fluorophore, et suivi par microscopie sa destination lorsqu'il est incubé avec des cellules dendritiques. Pf4 est absorbé par endocytose et est retrouvé co-localisé avec TLR3 dans les endosomes et les lysosomes. Ces résultats permettent aux auteurs de proposer la séquence d'évènements suivante: Pf4 est sécrété par $P$. aeruginosa dans le milieu extérieur, où il est capturé par des cellules phagocytaires, conduisant à l'activation de TLR3, ce qui induit la production d'IFN-I, qui inhibe la production de TNF- $\alpha$ et provoque une réduction de la phagocytose des bactéries. In fine, cette cascade aboutit à une diminution de la clairance de $P$. aeruginosa par le système immunitaire, et a pour conséquence une colonisation bactérienne de l'hôte accrue (Figure 1).

Un vaccin contre $P$. aeruginosa ciblant le bactériophage Pf4

À partir de leurs résultats associant le bactériophage Pf4 à la virulence de $P$. aeruginosa, les auteurs ont proposé une approche inédite de vaccin contre le bactériophage Pf4, fondée sur la production d'anticorps dirigés contre la protéine principale de sa capside. Cette vaccination, testée chez la souris, permet de réduire de moitié le nombre de plaies infectées par $P$. aeruginosa, et in

vitro, d'augmenter le nombre de bactéries phagocytées. Ce résultat est très prometteur car il pourrait ouvrir la voie au développement d'un nouveau type de vaccin, qui ne serait pas dirigé contre la bactérie elle-même, mais contre les bactériophages filamenteux qu'elle produit. La pertinence de cette approche est confirmée par une étude récente qui montre que la prévalence des bactériophages filamenteux dans les souches de $P$. aeruginosa isolées de patients atteints de mucoviscidose (chez qui l'infection pulmonaire par cette bactérie est fréquente) est d'environ $30 \%$ [4].

\section{Implications pour la phagothérapie}

L'activation d'une réponse antivirale par les molécules d'ARN d'un bactériophage dont le génome est constitué d'ADN simple brin est un phénomène inédit. Elle montre clairement que certains bactériophages interagissent avec le système immunitaire. Mais, comme le soulignent les auteurs, cette découverte ne remet pas en cause la phagothérapie qui repose sur l'utilisation de bactériophages virulents à $A D N$ double brin comme agents antibacté-

$(\rightarrow)$ Voir la Synthèse de N. Dufour et L. Debarbieux, $m / s n^{\circ} 4$, riens [8] $(\rightarrow)$.

Les deux types de bactériophages sont différents, et les travaux évaluant l'effet sur le système immunitaire de traitements
Figure 1. La bactérie Pseudomonas aeruginosa utilise le bactériophage filamenteux Pf4 pour augmenter sa virulence. Certaines souches de $P$. aeruginosa produisent le bactériophage Pf4 (étape 1), qui est absorbé (par endocytose) par les cellules immunitaires (étape 2). Le récepteur TLR3 (Toll-like récepteur 3 ) détecte alors la présence de molécules d'ARN du phage, ce qui déclenche la production d'interférons de type I (étape 3), qui est secrété (étape 4) et provoque, par la liaison à son récepteur, une réduction de la phagocytose de P. aeruginosa (étape 5).

par les bactériophages virulents n'ont jamais mis en évidence de réponse antivirale. De plus, notre équipe a démontré que les bactériophages et le système immunitaire agissent de façon synergique dans l'élimination de

$P$. aeruginosa des poumons d'animaux infectés $[5,9](\rightarrow)$.

$(\rightarrow)$ Voir la Nouvelle de M.De Jode et al., $m / s n^{\circ} 4$, avril 2018, page 291

Il est donc probable que le mécanisme décrit par ces auteurs soit restreint à quelques bactériophages filamenteux [6, 7]. Faut-il conclure de cette étude que ces bactériophages filamenteux détournent le système immunitaire pour maintenir une densité de bactéries plus importante afin d'assurer leur propre subsistance, ou bien les bactéries utilisent-elles ces bactériophages comme des armes pour contrer le système immunitaire de l'hôte? Ces travaux témoignent en tout cas de notre connaissance très incomplète du rôle joué par les bactériophages qui, plus de 100 ans après leur découverte $[10](\rightarrow)$, nous réservent proba-

$(\rightarrow)$ Voir la Chronique génomique de B. Jordan, $m / s n^{\circ} 10$, blement encore bien octobre 2019, page 806 des surprises... $\diamond$

Pseudomonas aeruginosa uses its filamentous bacteriophages to manipulate the immune system

\section{LIENS D'INTÉRÊT}

Les auteurs déclarent n'avoir aucun lien d'intérêt concernant les données publiées dans cet article. 


\section{RÉFÉRENCES}

1. Sweere JM, Van Belleghem JD, Ishak $\mathrm{H}$, et al. Bacteriophage trigger antiviral immunity and prevent clearance of bacterial infection. Science 2019; 363.

2. Mai-Prochnow A, Hui JG, Kjelleberg S, et al. Big things in small packages: the genetics of filamentous phage and effects on fitness of their host. FEMS Microbiol Rev $2015 ; 39: 465-87$

3. Secor PR, Michaels LA, Smigiel KS, et al. Filamentous bacteriophage produced by Pseudomonas aeruginosa alters the inflammatory response and promotes noninvasive infection in vivo. Infect Immun 2017 ; 85 e00648-16.
4. Burgener EB, Sweere JM, Bach MS, et al. Filamentous bacteriophages are associated with chronic Pseudomonas lung infections and antibiotic resistance in cystic fibrosis. Sci Transl Med 2019 11(488). pii: eaau9748.

5. Roach DR, Leung CY, Henry M, et al. Synergy between the host immune system and bacteriophage is essential for successful phage therapy against an acute respiratory pathogen. Cell Host Microbe 2017 ; $22: 38-47$ e 4

6. Jonczyk-Matysiak $\varepsilon$, Lusiak-Szelachowska M, Klak $M$, et al. The effect of bacteriophage preparations on intracellular killing of bacteria by phagocytes. J Immunol Res $2015 ; 2015$ : 482863.
7. Bille $\varepsilon$, Meyer J, Jamet A, et al. A virulence-associated filamentous bacteriophage of Neisseria meningitidis increases host-cell colonisation. PLoS Pathog 2017; 13 : el006495.

8. Dufour N, Debarbieux L. La phagothérapie : une arme crédible face à l'antibiorésistance. Med Sci (Paris) 2017 ; 33 : 410-16.

9. De Jode M, Roach D, Debarbieux L. La synergie immunophage au cœur du succès de la phagothérapie pulmonaire Med Sci (Paris) 2018 ; 34 : 291-3.

10. Jordan B. Cent ans après, le retour de la phagothérapie? Med Sci (Paris) 2019; $35: 80$ 